\title{
Análise de metodologias de avaliação da sustentabilidade hidroambiental segundo BellagioSTAMP
}

A busca pelo desenvolvimento sustentável tem estimulado tanto a utilização de indicadores como ferramentas de mensuração, quanto a elaboração de modelos conceituais para construção de metodologias de avaliação da sustentabilidade. Considerando o BellagioSTAMP importante modelo conceitual para nortear a construção destas metodologias, este trabalho tem como objetivo analisar metodologias de avaliação da sustentabilidade hidroambiental segundo os Princípios do BellagioSTAMP. Para isso, foi interpretado o BellagioSTAMP, destacando-se os critérios essenciais em cada princípio, realizada uma análise comparativa entre seis metodologias de avaliação da sustentabilidade hidroambiental publicadas em periódicos entre 2008 e 2015, estabelecido o percentual de atendimento das metodologias ao BellagioSTAMP e sua classificação em: ruim, regular, bom ou ótimo. Esta pesquisa é classificada quanto aos objetivos como exploratória e descritiva tendo como base a pesquisa bibliográfica e a análise de conteúdo. De posse dos critérios essenciais, cada metodologia foi analisada buscando-se o atendimento ou não a cada critério para fins de comparação, bem como seu percentual de atendimento. Os principais resultados mostraram que nenhuma metodologia atendeu integralmente aos princípios do BellagioSTAMP, cinco obtiveram na classificação um nível bom e apenas uma atingiu o nível ótimo, com $77,08 \%$ de atendimento. Além disso, observou-se que a maioria das metodologias não atenderam aos Princípios 3 e 7 , da análise espaço-temporal e da ampla participação, respectivamente, demonstrando pouca preocupação com as gerações futuras e baixa relevância política. Estes resultados apresentam relevante contribuição buscando auxiliar de forma prática futuras pesquisas de avaliação de sustentabilidade a seguirem os princípios do BellagioSTAMP.

Palavras-chave: Desenvolvimento Sustentável; Avaliação da Sustentabilidade; BellagioSTAMP.

\section{Analysis of methodologies for the evaluation of hydroambiental sustainability according to BellagioSTAMP}

The search for sustainable development has stimulated both the use of indicators and measurement tools, as well as the elaboration of conceptual models for the construction of sustainability assessment methodologies. Considering the BellagioSTAMP important conceptual model to guide the construction of these methodologies, this work aims to analyze methodologies of evaluation of the environmental sustainability according to the BellagioSTAMP Principles. For this, the BellagioSTAMP was interpreted, highlighting the essential criteria in each principle, a comparative analysis was carried out between six methodologies of evaluation of the environmental sustainability published in periodicals between 2008 and 2015, establishing the percentage of attendance of the methodologies to BellagioSTAMP and its rating on: bad, regular, good or great. This research is classified as exploratory and descriptive based on bibliographic research and content analysis. Based on the essential criteria, each methodology was analyzed by whether or not each criterion was used for comparison purposes, as well as its percentage of attendance. The main results showed that no methodology fully met the principles of BellagioSTAMP, five obtained a good level in the classification and only one reached the optimal level, with $77.08 \%$ of attendance. In addition, it was observed that most methodologies did not meet Principles 3 and 7 , of spacetime analysis and broad participation, respectively, showing little concern for future generations and low political relevance. These results present a relevant contribution seeking to help in a practical way future researches of sustainability evaluation to follow the principles of BellagioSTAMP.

Keywords: Sustainable Development; Sustainability Assessment; BellagioSTAMP.

Topic: Desenvolvimento, Sustentabilidade e Meio Ambiente

Reviewed anonymously in the process of blind peer.

Geórgia Cristina de Sousa Oliveira

Universidade Federal de Campina Grande, Brasi

http://lattes.cnpq.br/0194822514174392

georgia.ufpe@bol.com.br

Rosires Catão Curi (iD

Universidade Federal de Campina Grande, Brasil

http://lattes.cnpq.br/7625445154639508

http://orcid.org/0000-0001-6924-4204

rosirescuri@yahoo.com.br
Received: 10/02/2018

Approved: 24/03/2018
Referencing this:

OLIVEIRA, G. C. S.; CURI, R. C.. Análise de metodologias de avaliação da sustentabilidade hidroambiental segundo BellagioSTAMP. Revista Ibero Americana de Ciências Ambientais, v.9, n.3, p.275-288, 2018. DOI: http://doi.org/10.6008/CBPC2179-6858.2018.003.0022 


\section{INTRODUÇÃO}

A crise ambiental que a terra atravessa atualmente tem incentivado pesquisas em diferentes áreas acerca de medidas de mitigação das consequências da ação humana sobre o meio ambiente. Dentre as diversas contribuições, está o processo de mensuração do desenvolvimento sustentável, considerado aquele que atende as necessidades das gerações presentes sem comprometer as futuras gerações de atenderem suas próprias necessidades. Observa-se que tal consideração depende de uma nova relação do ser humano com o meio ambiente, baseado na capacidade de carga do ecossistema, ou seja, baseado em seus limites naturais. A capacidade de carga imprime que toda atividade deve ter limites para que não sobrecarregue a capacidade de renovação do meio ambiente e uma das principais ferramentas para se mensurar o desenvolvimento sustentável de um território são os indicadores. Segundo Van Bellen (2006) os indicadores podem comunicar ou informar sobre o progresso em direção a uma meta, bem como deixar mais perceptível uma tendência ou fenômeno, sendo estes importantes componentes da avaliação do progresso em relação a uma meta complexa como o desenvolvimento sustentável.

Visando avaliar o desenvolvimento sustentável, foram construídos modelos conceituais para orientar qualquer instrumento para esta finalidade, os quais devem atender a, pelo menos, 10 princípios básicos denominados Princípios de Bellagio et al. (1997). Os princípios foram criados por um grupo de especialistas em avaliação, na Fundação Rockfeller, Bellagio - Itália em 1996. Tais princípios servem como guia para avaliar e melhorar a escolha, utilização, interpretação e comunicação dos indicadores, são eles: 1- Visão e objetivos orientadores; 2- Perspectiva holística; 3- Elementos essenciais; 4- Âmbito adequado; 5- Foco prático; 6 Abertura; 7- Comunicação eficaz; 8- Ampla participação; 9- Avaliação contínua e 10- Capacidade institucional. No entanto, buscando aprimorar esta análise, em 2009, uma nova equipe de especialistas, reexaminou estes conceitos, condensando-os em 8 Princípios para Avaliação e Medição da Sustentabilidade (o BellagioSTAMP), tornando mais clara sua implementação, são eles (BAKKES, 2012): 1- Orientar a visão; 2- Considerações essenciais; 3- Escopo apropriado; 4- Estrutura e indicadores; 5- Transparência; 6- Comunicação eficaz; 7Ampla participação e 8- Capacidade de renovação e continuidade.

Diante deste contexto, subentende-se que todas as ferramentas de avaliação do desenvolvimento sustentável, qualquer que seja o tema deve utilizar tais modelos conceituais para auxiliar na escolha de indicadores, metodologia e apresentação dos resultados ao público. Contudo, desde sua criação em 1996, não é comum a utilização destes princípios como norteadores da construção de metodologias de avaliação da sustentabilidade, em especial da sustentabilidade hidroambiental ou da gestão dos recursos hídricos, tendo como base a análise de publicações em periódicos nacionais e internacionais, entre 2008 e 2015. Neste sentido, o principal objetivo deste trabalho será analisar as metodologias de avaliação da sustentabilidade hidroambiental mais recentes, com base na adequação aos Princípios do BellagioSTAMP, visando identificar em tais princípios os critérios essenciais para orientar qualquer metodologia de avaliação futura. Para isso, os seguintes objetivos específicos deverão ser atendidos: Interpretar o BellagioSTAMP destacando os critérios de análise essenciais em cada princípio para avaliação da sustentabilidade; Realizar uma análise 
comparativa entre seis metodologias de avaliação da sustentabilidade hidroambiental publicadas em periódicos entre 2008 e 2015 segundo os Princípios do BellagioSTAMP; Estabelecer o percentual de atendimento das metodologias ao BellagioSTAMP. Tais objetivos visam evidenciar a importância da utilização de um modelo conceitual, o BellagioSTAMP, para formulação de metodologias de avaliação da sustentabilidade, dando ao processo e aos resultados um caráter científico, com respaldo internacional e como apoio as decisões em prol de um futuro sustentável.

\section{METODOLOGIA}

\section{Caracterização da pesquisa}

De acordo com Gil (2002), uma pesquisa é classificada com base em seu objetivo. Considerando o objetivo de analisar metodologias de avaliação da sustentabilidade hidroambiental segundo os Princípios do BellagioSTAMP, o tipo de pesquisa aqui empregada é exploratória e descritiva, tendo a primeira como finalidade proporcionar mais informação sobre o assunto a ser investigado, possibilitando sua definição e delineamento e a segunda, visando observar, registrar, analisar e ordenar os dados sem manipulá-los, isto é, sem a interferência do pesquisador (PRODANOV et al., 2013). Já com base nos procedimentos técnicos utilizados, é uma pesquisa bibliográfica, desenvolvida com base em material já elaborado, construído principalmente de livros e artigos científicos, utilizando - se da análise de conteúdo para investigação, que segundo Severino (2016) é uma metodologia de tratamento e análise de informações constantes de um documento, sob a forma de discursos em diferentes linguagens, buscando-se compreender o sentido manifesto ou oculto das comunicações. Quanto à abordagem do problema (PRODANOV et al., 2013), tem-se uma pesquisa de cunho quali-quantitativa, por respectivamente considerar o processo de análise e interpretação dos dados mais importantes que o produto, e por traduzir em números as informações para posterior classificação. A natureza é de pesquisa aplicada, objetivando gerar conhecimentos para aplicação prática e solução de problemas específicos.

Procedimentos metodológicos

Os Princípios do BellagioSTAMP - modelo conceitual para orientar a construção de ferramentas de avaliação da sustentabilidade - foram interpretados para determinação dos critérios essenciais a eles associados. Com a definição dos critérios essenciais, foi realizada uma seleção de estudos de avaliação da sustentabilidade hidroambiental ou dos recursos hídricos publicados em periódicos nacionais ou internacionais. Durante a pesquisa, foram obtidas seis publicações entre 2008 e 2015 sobre esta temática. De posse das publicações, foram realizadas leituras e fichamentos buscando identificar a adequação aos critérios essenciais estabelecidos nos princípios em questão. Com base nos dados, foram construídos quadros para apresentação do conteúdo por metodologia/autor. Concluída esta etapa, partiu-se para a abordagem quantitativa dos dados, na qual foi estabelecido o percentual de atendimento aos Princípios do BellagioSTAMP. Para tanto, identificou-se o percentual de atendimento a cada critério, e em seguida, com o saldo total, estipulou-se o percentual de atendimento geral, dado pela seguinte Equação: 
Percentual de atentimento geral $=\frac{\sum \mathrm{Pn}}{800} \mathrm{x} 100$

$\sum \mathrm{Pn}=$ Soma dos percentuais obtidos em cada princípio analisado por metodologia;

Para fins de análise, realizou-se ainda uma classificação das metodologias considerando quatro intervalos de classe: ruim (0\%-25\%), regular (26\%-50\%), bom (51\%-75\%) e ótimo (76\%-100\%).

\section{DISCUSSÃO TEÓRICA}

\section{Princípios de Bellagio}

Até a década de 90 os principais indicadores de medição do desenvolvimento foram os econômicos, exemplo: Produto Interno Bruto (PIB), PIB per capita (ALSHUWAIKHAT et al., 2002). A avaliação do PIB permitia que os governos acompanhassem o fluxo de bens e serviços na economia. Considerado de fácil comunicação, popularizou-se como indicador do bem-estar de uma nação (HARDI et al., 1997). Essa forma de medição do progresso foi identificada como uma das alavancas para a construção de um desenvolvimento não sustentável, dado a incapacidade do indicador em provocar medidas preventivas quanto ao meio ambiente (PINTÉR et al., 2012). Com o surgimento do conceito de desenvolvimento sustentável, veio à necessidade de avaliar o impacto do desenvolvimento econômico sobre o meio ambiente e a sociedade (ALSHUWAIKHAT et al., 2002). Por isso, em 1987, a Comissão Brundtland introduziu novas formas para medir o progresso, para além dos sinais econômicos, capturando o bem-estar social e dos ecossistemas. Tal mudança estava relacionada tanto ao conceito de desenvolvimento sustentável, quanto ao reconhecimento de que é preciso uma alteração na natureza das atividades humanas para que as gerações futuras também possam atender suas necessidades (HARDI et al., 1997).

A partir daí, como se tornou preocupação geral monitorar e avaliar o progresso rumo ao desenvolvimento sustentável, cogitou-se inicialmente elaborar uma lista de indicadores comuns para avaliação do progresso global. Contudo, dado as diferenças entre lugares e de valores culturais, um grupo de pesquisadores em avaliação, de cinco continentes, elaboraram segundo Alshuwaikhat et al. (2002), importantes diretrizes para construção de instrumentos de avaliação da sustentabilidade, qualquer que seja a preocupação. De acordo com Ko (2005), este grupo, organizado pelo Instituto Internacional para o Desenvolvimento Sustentável (IISD), se reuniu no Centro de Estudos e Conferências da Fundação Rockefeller em Bellagio, Itália, em 1996, e elaboraram os chamados Princípios de Bellagio, nos quais estabeleceram orientações para medir e avaliar o progresso rumo ao desenvolvimento sustentável.

Os princípios estabelecidos foram aprovados por unanimidade e servem de orientação para toda avaliação, incluindo: escolha e concepção de indicadores, interpretação e comunicação dos resultados e medição do progresso. Eles estão inter-relacionados e devem ser aplicados como um conjunto completo (HARDI et al., 1997). Ou seja, qualquer instrumento para avaliar a sustentabilidade deve atender a, pelo menos, 10 princípios básicos, denominados Princípios de Bellagio, que servem como guia para avaliar e melhorar a escolha, utilização, interpretação e comunicação de indicadores. Os Princípios em questão são: 1 - Visão e objetivos orientadores; 2 - Perspectiva holística; 3 - Elementos essenciais; 4 - Âmbito adequado; 
5 - Foco prático; 6 - Abertura; 7 - Comunicação eficaz; 8 - Ampla participação; 9 - Avaliação contínua; e 10

- Capacidade institucional.

\section{BellagioSTAMP}

Segundo Pintér et al. (2012) os Princípios de Bellagio se tornaram um ponto de referência amplamente citado para medir o desenvolvimento. Mas, novos desdobramentos em matéria de política, ciência, sociedade civil e tecnologia tornaram necessária à sua atualização. Os Princípios de Bellagio foram atualizados através de um grupo de dezenove especialistas, um terço dos que atuaram em 1996, Bakkes (2012). Semelhante ao processo de construção inicial - o grupo reuniu-se na Fundação Rockfeller, Bellagio, Itália em 2009, e utilizando os princípios originais como ponto de partida criou o BellagioSTAMP - Princípios para Avaliação e Medição da Sustentabilidade.

Devendo ser usado como um conjunto completo, o novo BellagioSTAMP inclui oito princípios: 1 - Visão orientadora; 2 - Considerações essenciais; 3 - Escopo adequado; 4 - Estrutura e indicadores; 5 - Transparência; 6 - Comunicação eficaz; 7 - Ampla participação; e 8 - Continuidade e capacidade. Para Bakkes (2012), a nova equipe Bellagio, criou uma nova lista de princípios considerada conceitualmente mais condensada, e mais facilmente aplicável. Isto porque, segundo Pintér et al. (2012) os princípios foram sucintamente reformulados, eliminando algumas das ambiguidades e duplicações que estavam presentes no conjunto original e também inserindo novos pontos de ênfase, com isso, o número de princípios foi reduzido de dez para oito. Conforme Pintér (2009) e Pintér et al. (2012) os novos princípios do BellagioSTAMP podem ser descritos e classificados 8.

\section{Princípio 1: Visão orientadora}

Avalia que o progresso rumo ao desenvolvimento sustentável deve ser guiado pelo objetivo de atender o bem-estar da população considerando a capacidade de suporte da biosfera, para sustentá-la para as gerações futuras, Pintér (2009) e Pintér et al. (2012). Neste sentido, antes de se avaliar o progresso, é preciso definir qual é a visão de desenvolvimento sustentável adotada para se identificar a preocupação ou não com as gerações futuras.

\section{Princípio 2: Considerações essenciais}

Nele, a avaliação do progresso rumo ao desenvolvimento sustentável deve considerar o sistema social, econômico e ambiental subjacente como um todo e as interações entre seus componentes incluindo questões relacionadas com a governança, além da dinâmica e interação entre as tendências atuais e futuras; riscos, incertezas de atividades que podem ter impacto além dos limites; implicações na tomada de decisões, incluindo compromissos e sinergias, Pintér (2009) e Pintér et al. (2012). Neste cenário, destaca-se a análise prioritária das dimensões do desenvolvimento sustentável e as relações entre elas. 


\section{Princípio 3: Âmbito adequado}

Neste, a avaliação do progresso rumo ao desenvolvimento sustentável deve adotar um horizonte de tempo apropriado para captar os efeitos das decisões políticas atuais e das atividades humanas, tanto a curto como a longo prazo e um âmbito geográfico adequado, Pintér (2009) e Pintér et al. (2012). Ou seja, a avaliação deve permitir a análise espaço-temporal dos indicadores para que se avalie os efeitos das ações tomadas.

\section{Princípio 4: Estrutura e indicadores}

Baseia a avaliação do progresso rumo ao desenvolvimento sustentável em um quadro conceitualestrutural que identifique os indicadores-chave para avaliar o progresso; métodos de medição normalizados, com vistas à comparabilidade e comparação dos valores dos indicadores de metas, de referência ou do intervalo, sempre que possível, Pintér (2009) e Pintér et al. (2012). Este princípio apresenta itens relevantes para construção do modelo, destacando o número de indicadores adequados, que aqui será entre 23 e 32 indicadores, já que segundo Tanguay, Rajaonson e Lefebvre (2010) é o número suficiente para cobrir as dimensões do desenvolvimento sustentável. Destaca ainda a necessidade de normalização dos indicadores para fins de comparação, bem como a comparação entre os resultados com base em valores normativos e do intervalo, para que se tenha visões da colocação do município ou região quanto aos valores de referência, e quanto ao seu entorno.

\section{Princípio 5: Transparência}

Neste princípio, a avaliação do progresso em direção ao desenvolvimento sustentável deve garantir que os dados, os indicadores e os resultados sejam acessíveis ao público; explicar as escolhas, hipóteses e incertezas dos resultados da avaliação; divulgar fontes de dados e métodos; e divulgar todas as fontes de financiamento e potenciais conflitos de uso, Pintér (2009) e Pintér et al. (2012). Em outras palavras, uma metodologia de avaliação deve apresentar dados, métodos e resultados que sejam acessíveis ao público, tanto na forma de obtenção dos dados, quanto na compreensão dos resultados.

\section{Princípio 6: Comunicação eficaz}

Entende-se que a avaliação em direção ao desenvolvimento sustentável deva utilizar linguagem clara; apresentar a informação de forma objetiva; utilizar ferramentas e gráficos visuais inovadores para auxiliar na interpretação; e disponibilizar os dados com todos os detalhes para que sejam aplicáveis, Pintér (2009) e Pintér et al. (2012). Com isso, percebe-se que a informação deva ser apresentada de forma sucinta e clara, através de resultados quantitativos, utilizando-se elementos gráficos, cores, mapas, entre outros, para melhor visualização e entendimento. 


\section{Princípio 7: Ampla participação}

A avaliação rumo ao desenvolvimento sustentável deve buscar refletir as opiniões do público, especialmente dos utilizadores da avaliação, tais como: as lideranças, satisfazendo as suas necessidades, a fim de reforçar a legitimidade e relevância dos indicadores, Pintér (2009) e Pintér et al. (2012). Tal princípio tem como base a gestão participativa, seja com a participação de representantes de instituições e/ou comunidades para que sejam identificadas as necessidades do público e assim construído um modelo de avaliação adequado, legítimo e relevante.

\section{Princípio 8: Continuidade e Capacidade}

A avaliação em direção ao desenvolvimento sustentável exige medição repetida; capacidade de resposta à mudança; investimento para desenvolver e manter o monitoramento; e aprendizagem contínua, Pintér (2009) e Pintér et al. (2012). Ou seja, a avaliação deve permitir o monitoramento contínuo daquilo que se quer avaliar, para que possa ser utilizada como instrumento de apoio a decisão.

\section{Interpretação do BellagioSTAMP}

Sinteticamente, os princípios foram aqui interpretados para sua operacionalização da seguinte forma: o Princípio 1 - sugere que a avaliação para o desenvolvimento sustentável deva seguir uma visão orientadora e que esteja preocupada com a capacidade de suporte da biosfera, subjetivamente com as futuras gerações; o Princípio 2 - resultante da condensação dos itens Perspectiva holística e Elementos essenciais dos Princípios de Bellagio originais - atual Considerações essenciais, evidencia a necessidade de abordar as dimensões social, ambiental, econômica e institucional e as interações entre elas para a citada avaliação; o Princípio 3 - denominado Âmbito adequado, busca captar os efeitos das decisões políticas e das atividades humanas através de uma análise espaço-temporal apropriada como auxílio a tomada de decisão; no Princípio 4 -Estrutura e indicadores, consideram essencial a utilização de indicadores chave, padronização de medidas e a comparação entre os valores de referência ou normativos e os valores do intervalo, para tornar aplicável a ferramenta, tanto na análise entre territórios quanto na análise individual; o Princípio 5 Transparência, solicita que dados e métodos sejam respectivamente acessíveis e detalhados para que o público possa entendê-los e utilizá-los; no Princípio 6 - a Comunicação eficaz busca apresentar de forma objetiva e didática os resultados obtidos, devendo utilizar para tanto, dados numéricos e recursos visuais adequados para auxiliar na interpretação; o Princípio 7 - Ampla participação, sugere que na construção de modelos de avaliação sejam consideradas as opiniões do público para reforçar a legitimidade e relevância dos indicadores.

Segundo Van Bellen (2006), dois tipos de abordagem podem ser utilizados: a top-down e a bottomup, a primeira considera apenas a opinião de especialistas e a segunda, inicia com o posicionamento dos decisores e conclui com a análise de especialistas, sendo que a ocorrência de uma ou outra não invalida o resultado. 0 último princípio, o 8, resultado da junção dos itens 9 e 10 do Bellagio de 1996, exige que a 
avaliação possa ser repetida para fins de monitoramento e que seja sobretudo adaptável as mudanças, com a inserção ou exclusão de indicadores que sejam adequados à gestão.

\section{Comparação das metodologias de sustentabilidade hídrica segundo o BellagioSTAMP}

De posse dos dados (Quadro 1), observa-se que Guimarães et al. (2008), Vieira et al. (2009) e Carvalho et al. (2015) apresentam definições de desenvolvimento sustentável que orientam a pesquisa, seja buscando a manutenção do bem-estar ou melhoria das condições de vida da população atual e das gerações futuras, ou baseada no conceito de sustentabilidade hidroambiental porém, nenhuma considera a capacidade de suporte da biosfera. Já Francisco et al. (2008), Pereira et al. (2012), Sales et al. (2013) são orientados por uma visão de desenvolvimento sustentável que objetiva melhorar a qualidade de vida humana dentro dos limites da capacidade de suporte dos ecossistemas.

Entendendo capacidade de suporte como: "a capacidade de um ecossistema manter seus organismos saudáveis e, ao mesmo tempo, manter sua produtividade, adaptabilidade e capacidade de renovação" UICN et al. (1991 citado por FRANCISCO et al., 2008). Tal visão coaduna com a orientação do BellagioSTAMP, que destaca a necessidade de considerar a capacidade de suporte da biosfera quando se trabalha com desenvolvimento sustentável.

Quadro 1: Visão orientadora.

\begin{tabular}{|l|l|l|}
\hline Autores/metodologia & Visão de Desenvolvimento Sustentável & Capacidade de suporte da biosfera \\
\hline Guimarães et al. (2008) & Sim & Não \\
\hline Francisco et al. (2008) & Sim & Sim \\
\hline Vieira et al. (2009) & Sim & Não \\
\hline Pereira et al. (2012) & Sim & Sim \\
\hline Sales et al. (2013) & Sim & Sim \\
\hline Carvalho et al. (2015) & Sim & Não \\
\hline
\end{tabular}

As metodologias desenvolvidas por Francisco et al. (2008), Vieira et al. (2009), Carvalho et al. (2015), levando-se em consideração os indicadores utilizados (Quadro 2), consideraram as dimensões social, econômica e ambiental do desenvolvimento sustentável, exceto a institucional. Deste modo, como não há indicadores institucionais na questão, não podemos dizer que atende ao Princípio 2 do BellagioSTAMP. Por outro lado, Guimarães et al. (2008), Pereira et al. (2012), Sales et al. (2013) utilizaram conceitos que permitem a análise das quatro dimensões do desenvolvimento sustentável indicados pela Comissão de Desenvolvimento Sustentável da Organização das Nações Unidas (CDS - ONU), são elas: dimensões social, econômica, ambiental e institucional, e suas inter-relações com os recursos hídricos.

Quadro 2: Considerações essenciais.

\begin{tabular}{|l|l|l|l|l|}
\hline \multirow{2}{*}{ Autores/metodologia } & \multicolumn{4}{|l|}{ Dimensões do Desenvolvimento Sustentável } \\
\cline { 2 - 5 } & Social & Ambiental & Econômica & Institucional \\
\hline Guimarães et al. (2008) & Sim & Sim & Sim & Sim \\
\hline Francisco et al. (2008) & Sim & Sim & Sim & Não \\
\hline Vieira et al. (2009) & Sim & Sim & Sim & Não \\
\hline Pereira et al. (2012) & Sim & Sim & Sim & Sim \\
\hline Sales et al. (2013) & Sim & Sim & Sim & Sim \\
\hline Carvalho et al. (2015) & Sim & Sim & Sim & Não \\
\hline
\end{tabular}


Com base nos dados sobre Escopo adequado (Quadro 3), vê-se que todas as metodologias aqui representadas, não realizaram avaliações espaço-temporal visando identificar tendências, exceto Vieira et al. (2009) que realizaram uma análise comparativa da região da Área de Proteção Ambiental Baturité/CE, por dois períodos distintos (2005 e 2013), atendendo, portanto, ao Princípio 3 do modelo conceitual em análise.

Quadro 3: Escopo adequado.

\begin{tabular}{|l|l|}
\hline Autores/metodologia & Análise espaço-temporal \\
\hline Guimarães et al. (2008) & Não \\
\hline Francisco et al. (2008) & Não \\
\hline Vieira et al. (2009) & Sim \\
\hline Pereira et al. (2012) & Não \\
\hline Sales et al. (2013) & Não \\
\hline Carvalho et al. (2015) & Não \\
\hline
\end{tabular}

Com base nos dados sobre Estrutura e indicadores (Quadro 4), verifica-se que as metodologias de Vieira et al. (2009), Sales et al. (2013) preocuparam-se em selecionar indicadores-chave e em número limitado, entre 23 e 32 indicadores que segundo Tanguay et al. (2010) são capazes de cobrir as dimensões do desenvolvimento sustentável, padronizaram as medidas, permitindo a comparação entre os dados, no entanto, não realizaram análise entre os resultados reais ou do intervalo, com os de referência (normativos), para avaliação do comportamento dos dados em diferentes perspectivas. Já Francisco et al. (2008) e Carvalho et al. (2015), apesar de terem padronizado as medidas, apresentaram ora um pequeno, ora um grande número de indicadores-chave, não atendendo ao número ideal de indicadores. Além disso, assim como os anteriores, também não realizaram a comparação dos resultados entre os valores do intervalo (dados reais) com os normativos (de referência) para verificar o comportamento dos indicadores.

Neste sentido, a única metodologia que atende ao Princípio 4 do BellagioSTAMP é a de Guimarães et al. (2008), na qual foi apresentado um quadro de indicadores de sustentabilidade para bacias hidrográficas, com um número limitado de indicadores (totalizando 32 indicadores); padronização das medidas e a possibilidade de comparação entre os indicadores do intervalo e os indicadores de referência pois apresenta valores normativos para análise. Contrariamente, a metodologia utilizada por Pereira et al. (2012) por não ter em sua estrutura um número limitado de indicadores, ou seja, entre 23 e 32 indicadores, não terem padronizado as medidas para fins de comparação e não terem comparado os resultados dos níveis de referência com os do intervalo, para verificar além da condição geral, o quanto cada município está mais desenvolvido que o outro, foi o único que não atendeu a este princípio em nenhum aspecto.

Quadro 4: Estrutura e indicadores.

\begin{tabular}{|l|c|c|l|}
\hline Autores/metodologia & $\begin{array}{l}\text { № limitado de } \\
\text { indicadores }\end{array}$ & $\begin{array}{l}\text { Padronização } \\
\text { medidas }\end{array}$ & $\begin{array}{l}\text { Comparação entre indicadores do intervalo e de } \\
\text { referência }\end{array}$ \\
\hline Guimarães et al. (2008) & 32 & Sim & Sim \\
\hline Francisco et al. (2008) & 4 & Sim & Não \\
\hline Vieira et al. (2009) & 27 & Sim & Não \\
\hline Pereira et al. (2012) & 299 & Não & Não \\
\hline Sales et al. (2013) & 32 & Sim & Não \\
\hline Carvalho et al. (2015) & 46 & Sim & \\
\hline
\end{tabular}

Quanto ao quesito Transparência (Quadro 5), Francisco et al. (2008), Pereira et al. (2012), Sales et al. 2013) apresentaram as metodologias detalhadamente, porém os dados não foram considerados aqui de fácil 
acesso ao público, seja porque não foram demonstradas as fontes dos mesmos, ou quando apresentadas foram obtidas em fontes primárias, sendo os dados primários de difícil monitoramento - por serem dados não-oficiais e de consulta direta, ou porque para sua utilização necessita profissionais especializados para entendê-los e utilizá-los, dificultando assim a replicação, sobretudo como apoio a gestão. Todavia, Guimarães et al. (2008), Vieira et al. (2009) e Carvalho et al. (2015) preocuparam-se com a escolha dos indicadores, buscando utilizar dados oficiais e em fontes disponíveis, portanto, acessíveis ao público, aliado a isto apresentaram detalhadamente as metodologias desenvolvidas atendendo ao Princípio 5 das orientações BellagioSTAMP. Segundo Guimarães et al. (2008), tal escolha visa reduzir custos, permitir a reprodução e gerar confiabilidade.

Quadro 5: Transparência.

\begin{tabular}{|l|l|l|}
\hline Autores/metodologia & Dados acessíveis & Metodologia detalhada \\
\hline Guimarães et al. (2008) & Sim & Sim \\
\hline Francisco et al. (2008) & Não & Sim \\
\hline Vieira et al. (2009) & Sim & Sim \\
\hline Pereira et al. (2012) & Não & Sim \\
\hline Sales et al. (2013) & Não & Sim \\
\hline Carvalho et al. (2015) & Sim & Sim \\
\hline
\end{tabular}

No tocante ao Princípio da Comunicação eficaz (Quadro 6), pode-se dizer que as metodologias de Guimarães et al. 2008), Francisco et al. (2008), Vieira et al. (2009), Sales et al. (2013) e Carvalho et al. (2015) apresentaram os resultados de forma numérica, portanto objetiva, e a estes estavam relacionados alguma representação visual, seja através de mapas temáticos, por classificação e performances em diferentes cores, ou simplesmente através de gráficos, em especial os do tipo radar/ameba visando maior esclarecimento para o público alvo. Contudo, a única metodologia que atendeu parcialmente a este quesito foi a de Pereira et al. (2012) na qual os resultados são apresentados utilizando-se ferramentas visuais, tais como: cores para ilustrar o nível de sustentabilidade dos indicadores, mas os resultados não são apenas numéricos, portanto não objetivos.

Quadro 6: Comunicação eficaz.

\begin{tabular}{|l|l|l|}
\hline Autores/metodologia & Resultados objetivos (numéricos) & Ferramentas visuais para apresentação \\
\hline Guimarães et al. (2008) & Sim & Sim \\
\hline Francisco et al. (2008) & Sim & Sim \\
\hline Vieira et al. (2009) & Sim & Sim \\
\hline Pereira et al. (2012) & Não & Sim \\
\hline Sales et al. (2013) & Sim & Sim \\
\hline Carvalho et al. (2015) & Sim & Sim \\
\hline
\end{tabular}

No Princípio da Ampla participação (Quadro 7) é considerado essencial atender as necessidades do público, o que torna a ferramenta relevante para a gestão e legítima, por atender as expectativas da sociedade. Neste sentido, as metodologias foram analisadas tanto no processo de participação, quanto no tipo de abordagem: top-down ou bottom-up. Sendo na primeira considerada apenas a participação de especialistas, e na segunda, o envolvimento do público interessado e especialistas concomitantemente. Neste sentido, observa-se que entre as seis metodologias analisadas, apenas duas aplicaram o critério da participação na seleção de indicadores, foram às metodologias utilizadas por Pereira et al. (2012) e Sales et 
al. (2013). Todavia, embora tenham levado em consideração a participação, não foi aplicada nenhuma das abordagens citadas por Van Bellen (2006), assim como nas demais metodologias.

Quadro 7: Ampla participação.

\begin{tabular}{|l|l|l|l|}
\hline \multirow{2}{*}{ Autores/metodologia } & \multirow{2}{*}{ Processo participativo } & \multicolumn{2}{l|}{ Abordagem } \\
\cline { 3 - 4 } & & Top - down & Bottom-up \\
\hline Guimarães et al. (2008) & Não & - & - \\
\hline Francisco et al. (2008) & Não & - & - \\
\hline Vieira et al. (2009) & Não & - & - \\
\hline Pereira et al. (2012) & Sim & - & - \\
\hline Sales et al. (2013) & Sim & - & - \\
\hline Carvalho et al. (2015) & Não & - & - \\
\hline
\end{tabular}

O Princípio Continuidade e capacidade (Quadro 8) destaca duas questões essenciais: a possibilidade de monitoramento dos dados pela gestão para fins de comparação e verificação de mudanças ao longo do tempo; e a possibilidade de adaptação dos indicadores em virtude de atualizações políticas, sociais, ambientais ou econômicas. Neste sentido, verificando-se os dados utilizados e as declarações dos autores sobre a importância das metodologias desenvolvidas, pode-se destacar que Guimarães et al. (2008), Francisco et al. (2008), Vieira et al. (2009), Sales et al. (2012) e Carvalho et al. (2015), atendem integralmente ao Princípio 8 do BellagioSTAMP, pois são ferramentas que podem ser reproduzidas, embora algumas com maior dificuldade por exigirem conhecimentos técnicos ou especializados. No entanto, apenas a metodologia utilizada por Pereira et al. (2012) teria difícil aplicação especialmente pela busca de dados das 299 variáveis, muitas vezes não publicados por órgãos oficiais, mesmo está atendendo à necessidade de adaptação às mudanças.

Quadro 8: Continuidade e capacidade.

\begin{tabular}{|l|l|l|}
\hline Autores/metodologia & Monitoramento contínuo & É adaptável às mudanças \\
\hline Guimarães et al. (2008) & Sim & Sim \\
\hline Francisco et al. (2008) & Sim & Sim \\
\hline Vieira et al. (2009) & Sim & Sim \\
\hline Pereira et al. (2012) & Não & Sim \\
\hline Sales et al. (2013) & Sim & Sim \\
\hline Carvalho et al. (2015) & Sim & Sim \\
\hline
\end{tabular}

\section{Percentual de atendimento das metodologias ao BellagioSTAMP}

Para obtenção deste resultado, em cada princípio foi estabelecido uma distribuição percentual conforme os critérios de análise essenciais definidos (Tabela 1). Assim, pode-se observar que para cada critério atendido um valor percentual está relacionado. E o resultado final consistirá no somatório do resultado por metodologia/autor, multiplicado por 100 e dividido por 800 (nota máxima), obtendo-se, portanto, os valores percentuais gerais.

Assim, considerando o percentual de atendimento aos Princípios do BellagioSTAMP (Tabela 2), observa-se que a metodologia que obteve o melhor resultado foi a de Sales et al. (2013), com 77,08\% de atendimento, seguida por Vieira et al. (2009) com 73,95\%. O terceiro lugar ficou para Guimarães et al. (2008) com 68,75\% de atendimento aos princípios, a quarta colocação ficou entre Francisco et al. (2008) e Carvalho et al. (2015), ambos com 61,45\% de atendimento aos itens analisados, e o último lugar ficou com Pereira et 
al. (2012), que respondeu a apenas 56,25\% dos itens, devido especialmente a grande quantidade de indicadores utilizados (299).

Tabela 1: Critérios essenciais do BellagioSTAMP e distribuição percentual.

\begin{tabular}{|c|c|c|c|c|c|}
\hline Princípios & \multicolumn{4}{|c|}{ Critérios essenciais - distribuição percentual } & \multirow{2}{*}{$\begin{array}{l}\begin{array}{l}\text { Total } \\
\text { (\%) }\end{array} \\
100 \\
\end{array}$} \\
\hline 1 & \multicolumn{2}{|c|}{ Visão de desenvolvimento sustentável (50\%) } & \multicolumn{2}{|c|}{ Capacidade de suporte da biosfera (50\%) } & \\
\hline 2 & Dimensão social (25\%) & $\begin{array}{l}\text { Dimensão ambiental } \\
(25 \%)\end{array}$ & $\begin{array}{l}\text { Dimensão } \\
\text { econômica (25\%) }\end{array}$ & $\begin{array}{l}\text { Dimensão } \\
\text { institucional (25\%) }\end{array}$ & 100 \\
\hline 3 & \multicolumn{4}{|c|}{ Análise espaço temporal (100\%) } & 100 \\
\hline 4 & $\begin{array}{l}\text { № de indicadores entre } \\
23 \text { e } 32(33,3 \%)\end{array}$ & $\begin{array}{l}\text { Padronização } \\
\text { medidas }(33,3 \%)\end{array}$ & \multicolumn{2}{|c|}{$\begin{array}{l}\text { Comparação entre valores normativos e do } \\
\text { intervalo }(33,3 \%)\end{array}$} & 100 \\
\hline 5 & \multicolumn{2}{|c|}{ Metodologia detalhada (50\%) } & \multicolumn{2}{|c|}{ Dados acessíveis (50\%) } & 100 \\
\hline 6 & \multicolumn{2}{|c|}{ Resultados quantitativos (50\%) } & \multirow{2}{*}{\multicolumn{2}{|c|}{ Representação gráfica (50\%) }} & 100 \\
\hline 7 & \multicolumn{2}{|l|}{ Participação pública (100\%) } & & & 100 \\
\hline 8 & \multicolumn{2}{|c|}{ Monitoramento contínuo (50\%) } & \multicolumn{2}{|c|}{ Adaptável as mudanças (50\%) } & 100 \\
\hline
\end{tabular}

Ainda de acordo com os dados percentuais, em caso de classificação das metodologias e considerando quatro classes, a saber: ruim (0\%-25\%), regular (26\%-50\%), bom (51\%-75\%), ótimo (76\%$100 \%)$, pode-se dizer que nenhuma das metodologias estão inseridas nas classes ruim ou regular, que cinco foram consideradas com um bom nível e apenas uma chegou ao nível ótimo, a de Sales e Cândido (2013). Vale ressaltar que esta análise não invalida nenhuma das metodologias desenvolvidas, apenas destaca lacunas que se atendidas, respondem as expectativas pretendidas para uma ferramenta de avaliação da sustentabilidade conforme orienta o BellagioSTAMP.

Tabela 2: Dados do percentual de atendimento ao BellagioSTAMP por princípio e total.

\begin{tabular}{|l|l|l|l|l|l|l|l|l|l|l|}
\hline $\begin{array}{l}\text { Autores/ } \\
\text { Metodologia }\end{array}$ & $\mathbf{P 1}$ (\%) & $\begin{array}{l}\text { P2 } \\
\text { (\%) }\end{array}$ & P3 (\%) & P4 (\%) & P5 (\%) & P6 (\%) & P7 (\%) & P8 (\%) & Total & \% de atendimento \\
\hline Guimarães et al. (2008) & 50 & 100 & 0 & 100 & 100 & 100 & 0 & 100 & 550 & 68,75 \\
\hline Francisco et al. (2008) & 100 & 75 & 0 & 66,6 & 50 & 100 & 0 & 100 & 491,6 & 61,45 \\
\hline Vieira et al. (2009) & 50 & 75 & 100 & 66,6 & 100 & 100 & 0 & 100 & 591,6 & 73,95 \\
\hline Pereira et al. (2012) & 100 & 100 & 0 & 0 & 50 & 50 & 100 & 50 & 450 & 56,25 \\
\hline Sales et al. (2013) & 100 & 100 & 0 & 66,6 & 50 & 100 & 100 & 100 & 616,6 & 77,08 \\
\hline Carvalho et al. (2015) & 50 & 75 & 0 & 33,3 & 100 & 100 & 0 & 100 & 491,6 & 61,45 \\
\hline
\end{tabular}

\section{CONCLUSÕES}

Os principais resultados mostram que todas as metodologias apresentaram uma visão norteadora sobre desenvolvimento sustentável, porém, 50\% não levou em consideração a capacidade de suporte da biosfera. Por outro lado, todas as metodologias atenderam pelo menos três dimensões do desenvolvimento sustentável a saber: social, econômica e ambiental, contudo, apenas 50\% conseguiram inserir aspectos políticos-institucionais como propõe a Comissão de Desenvolvimento Sustentável da ONU. Quanto à análise espaço-temporal percebeu-se que não é comum ser aplicada, visto que apenas Vieira et al. (2009) a realizaram. No tocante ao Princípio 4 - Estrutura e indicadores -, observa-se que a maioria buscou utilizar um número limitado de indicadores, bem como padronizar as medidas para fins de comparação, no entanto, percebe-se que não foram realizadas análises entre valores do intervalo e valores normativos, já que um município pode estar mais desenvolvido em comparação ao outro, porém considerando valores normativos, isto pode não acontecer. 
Todas as metodologias foram detalhadamente apresentadas, porém apenas $50 \%$ apresentaram fontes dos dados acessíveis, sendo que a metodologia de Pereira et al. (2012) além de não utilizar dados de fácil acesso ao público pois, consideraram diferentes fontes para obtenção (oficiais e não-oficiais), possuem um número de indicadores que extrapola os limites desejáveis. Pode-se dizer que todas as metodologias apresentaram seus resultados através de algum recurso visual (gráfico, mapa temático, classificação por cores), e de forma objetiva, ou seja, com respostas quantitativas, exceto a metodologia de Pereira et al. (2012) que utilizou dados quanti-qualitativos. Poucas metodologias adotaram o processo participativo em sua construção, apenas Sales et al. (2013) e Pereira et al. (2012), com a participação dos atores locais.

O Princípio 8 da Continuidade e capacidade foi atendido na íntegra por todas as metodologias, exceto, Pereira et al. (2012) especialmente pela dificuldade de monitoramento dos 299 indicadores utilizados. Através da classificação das metodologias, pode-se perceber que nenhuma apresentou resultados entre ruim e regular, pelo contrário, a maioria obteve um nível bom e apenas uma o nível ótimo, com 77,08\% de atendimento, que foi a metodologia de Sales et al. (2013).

Contudo, vale ressaltar que nenhuma atendeu integralmente aos Princípios do BellagioSTAMP, e no tocante aos Princípios 3 e 7, da análise espaço temporal e da ampla participação respectivamente, os mesmos obtiveram os menores valores de atendimento, demonstrando falha com a análise de tendência, ou seja, com as futuras gerações e pouca legitimidade e relevância política, haja vista a falta de participação do público alvo, especialmente dos decisores. Todo este esforço de análise evidenciou os critérios essenciais para construção de diversas metodologias de avaliação da sustentabilidade servindo, portanto, como orientação para pesquisas futuras em torno desta temática.

\section{REFERÊNCIAS}

ALSHUWAIKHAT, H. M.; NKWENTI, D. I.. Developing Sustainable Cities in Arid Regions. Cities, v.19, n.2, p.85-94, 2002.

BAKKES, J.. Bellagio Sustainability Assessment and Measurement Principles (Bellagio Stamp) - Significance and examples from internacional environment outlooks. In: VON RAGGAMBY, A.; RUBIK, F.. Sustainable Development Evoluation and Policy-Making: Theory, practice and quality Assurance. Cheltenham: Edward Elgar, 2012.

CARVALHO, J. R. M.;CÂNDIDO, G. A.; CURI, W. F.;ARAÚJO, E. K. M.. Análise da Sustentabilidade Hidroambiental de Municípios da Região da Sub-Bacia do Alto Piranhas, PB. Revista Holos, v.6, n.31, 2015.

FRANCISCO, C. N.; CARVALHO, C. N.. Avaliação da Sustentabilidade Hídrica de Municípios Abastecidos por Pequenas Bacias Hidrográficas: o caso de Angra dos Reis, RJ. Revista Brasileira de Recursos Hídricos, v.13, n.2, p.15-30, 2008.

GIL, A. C.. Como elaborar projetos de pesquisa. 4 ed. São Paulo: Atlas, 2002.

GUIMARÃES, L. T.; MAGRINI, A.. A proposal of indicators for sustainable development in the management of river basins.
Water Resour Manage, v.22, n.9, p.1191-1202, 2008. DOI: http://doi.org/10.1007/s11269-007-9220-x

HARDI, P.; ZDAN, T.. Principles in Pratice development: principles in pratice. Winnipeg: IISD, 1997.

KO, T. G.. Development of a tourism sustainability assessment procedure: a conceptual approach. Tourism Management, v.26, n.3, p.431-445, 2005.

PEREIRA, R. M. P. G.; CÂNDIDO, G. A.. Análise da Sustentabilidade da Gestão dos Recursos Hídricos: Um Estudo Exploratório Na Região da Bacia do Alto Curso do Rio Paraíba/PB. Revista Verde de Agroecologia e Desenvolvimento Sustentável, 2012.

PINTÉR, L.. Bellagio STAMP: Sustainability Assessment and measurement principles. In: WORLD FORUM ON MEASURING THE PROGRESS OF SOCIETIES, 3. Anais. Busan, 2009 .

PÍNTER, L.; HARDI, P.; MARTINUZZI, A.; HALL, J.. Bellagio Stamp: principles for sustainability assessment and measurement. Ecological indicators, v.17, p.20-28, 2012. 
PRODANOV, C. C.; FREITAS, E. C.. Metodologia do Trabalho Científico: Métodos e técnicas de Pesquisa e do Trabalho Acadêmico. Novo Hamburgo: FEEVALE, 2013.

SALES, L. G. L.; CÂNDIDO, G. A.. Análise da sustentabilidade hidroambiental dos municípios pertencentes a sub-bacia do Rio do Peixe/PB. Revista Verde de Agroecologia e Desenvolvimento Sustentável, v.8, n.5, p.22-40, 2013.

TANGUAY, G. A.; RAJAONSON, J.; LEFEBVRE, J. F.; LANOIE, P.. Measuring the sustainability of cities: can analysis of the use of local indicators. Ecological Indicators, v.10, n.2, p.407418, 2010.

VAN BELLEN, H. M.. Indicadores de Sustentabilidade. Rio de Janeiro: FGV, 2006.

VIEIRA, P. M. S.; STUDART, T. M. C.. Proposta Metodológica para o Desenvolvimento de um Índice de Sustentabilidade Hidro- Ambiental de Áreas Serranas no Semiárido Brasileiro Estudo de Caso: Maciço de Baturité, Ceará. Revista Brasileira de Recursos Hídricos, v.14, n.4, p.125-136, 2009.

A CBPC - Companhia Brasileira de Produção Científica (CNPJ: 11.221.422/0001-03) detém os direitos materiais desta publicação. Os direitos referem-se à publicação do trabalho em qualquer parte do mundo, incluindo os direitos às renovações, expansões e disseminações da contribuição, bem como outros direitos subsidiários. Todos os trabalhos publicados eletronicamente poderão posteriormente ser publicados em coletâneas impressas sob coordenação da Sustenere Publishing, da Companhia Brasileira de Produção Científica e seus parceiros autorizados. Os (as) autores (as) preservam os direitos autorais, mas não têm permissão para a publicação da contribuição em outro meio, impresso ou digital, em português ou em tradução. 